\title{
Análise crítica das recomendações da Associação Americana de Diabetes para doença cardiovascular no diabetes melito
}

\author{
Critical analysis of the American Diabetes Association dietary \\ recommendations for cardiovascular disease in diabetes mellitus
}

Ana Luiza Teixeira dos Santos' ${ }^{1}$, Tanara Weiss ${ }^{1}$, Camila Kümmel Duarte' Mirela J. de Azevedo', Themis Zelmanovitz ${ }^{1}$

\section{RESUMO}

A doença cardiovascular (DCV) é a principal causa de mortalidade em pacientes com diabetes melito (DM), sendo essencial a intervenção dietética no manejo dessa complicação. 0 objetivo deste manuscrito foi revisar as evidências científicas que fundamentam as recomendações dietéticas da American Diabetes Association (ADA) para prevenção e tratamento da DCV nos pacientes com DM. As diretrizes da ADA baseiam-se, em sua maioria, em estudos com pacientes com DCV, porém sem DM. Nos pacientes com DM, um aumento na ingestão de peixe e de fibras solúveis são as recomendações dietéticas com benefício comprovado. Embora o DM possa ser considerado um equivalente de DCV estabelecida, a adoção das recomendações dietéticas de pacientes sem DM e com DCV para todos pacientes com DM é questionável - em especial quando são consideradas as peculiaridades da DCV no DM. Ensaios clínicos aleatorizados em pacientes com DM deverão fundamentar melhor os benefícios das intervenções dietéticas sobre a DCV. Arq Bras Endocrinol Metab. 2009;53(5):657-66.

Descritores

Diabetes melito; doença cardiovascular; fatores de risco; intervenção dietética

\begin{abstract}
Cardiovascular disease (CVD) is the main cause of mortality among patients with diabetes mellitus (DM), and dietary intervention is an essential measure to prevent and treat this complication. The aim of this manuscript was to review scientific evidence that underlies the dietetic recommendations of the American Diabetes Association (ADA) for prevention and treatment of CVD in patients with DM. The ADA guidelines are mostly based on studies performed on patients with CVD and without DM. The evidence-based dietary recommendations for patients with DM are to increase the intake of fish and soluble fibers. Although DM has been considered as an equivalent of established CVD, the adoption of the same dietary recommendations for patients without DM and with CVD for all patients with DM is still questionable - especially considering the peculiarities of CVD in DM. Randomized clinical trials including patients with DM should provide further information regarding the benefits of these dietary interventions for CVD. Arq Bras Endocrinol Metab. 2009;53(5):657-66.
\end{abstract}

Keywords

Diabetes mellitus; cardiovascular disease; risk factors; dietetic intervention

\section{INTRODUÇÃO}

$\Delta^{2}$ doença cardiovascular (DCV) é a principal causa de morbidade e mortalidade em pacientes com diabetes melito (DM) (1), destacando-se, em especial, a
${ }^{1}$ Serviço de Endocrinologia, Hospital de Clínicas de Porto Alegre, Universidade Federal do Rio Grande do Sul (UFRGS) Porto Alegre, RS, Brasil

\author{
Correspondência para: \\ Themis Zelmanovitz \\ Serviço de Endocrinologia, Hospital \\ de Clínicas de Porto Alegre \\ Rua Ramiro Barcelos, 2.350, prédio \\ $12,4 \circ$ andar \\ 90035-003 - Porto Alegre, RS ,Brasil \\ themis.voy@terra.com.br
}

Recebido em 29/Mar/2009

Aceito em 29/Jun/2009 
crovasculares nestes pacientes equivalem a duas vezes as complicações microvasculares (2). Estudo transversal com 927 pacientes com DMT2 atendidos em nível ambulatorial em três centros médicos do Rio Grande do Sul observou uma prevalência de doença arterial coronariana de $36 \%$, doença arterial periférica de $33 \%$ e hipertensão de $73 \%$ (3). A coronariopatia, sobretudo, incide mais frequentemente e de maneira mais grave nos pacientes com DM em relação a pacientes não diabéticos (4). Essa condição é, em grande parte, explicada pela maior prevalência dos fatores de risco tradicionais entre os pacientes com DM (5), entre eles a obesidade, a dislipidemia e a hipertensão. De fato, a síndrome metabólica, condição sabidamente associada ao maior risco de DCV (6), está presente em mais do que $80 \%$ dos pacientes com DMT2, independente do critério utilizado como definição (7).

As medidas de prevenção da DCV nos pacientes com DM visam especialmente reverter os fatores de risco modificáveis (8), como abordagens sobre o estilo de vida. Entre as intervenções, destaca-se o manejo da dieta. A intervenção dietética se baseia principalmente nas características de alguns micro e macronutrientes, protetoras ou promotoras das complicações cardiovasculares (9). Essas características têm sido definidas com base em estudos epidemiológicos e experimentais em indivíduos sem DM. As dietas têm sido definidas como protetoras quando ricas em fibras e pobres em alimentos com carboidratos simples refinados $(10)$ e em alimentos processados comercialmente e com baixo teor de sal (11). São descritos, ainda, como nutrientes protetores o ácido graxo (AG) oleico, ácido linoleico, os AG poli-insaturados (AGPI) $\omega 3$, folato, vitamina B12 e vitaminas antioxidantes (betacaroteno, vitamina $\mathrm{C}$ e vitamina $\mathrm{E})(11,12)$. Entre os nutrientes considerados como promotores de DCV, estão o colesterol, os AG saturados (AGS), os AG insaturados trans e o conteúdo excessivo de sódio (13).

Nas diretrizes da ADA, nas quais as intervenções de dieta para o DM são revisadas $(14,15)$, as recomendações dietéticas para a DCV são de caráter geral. Recomendam-se uma dieta rica em frutas, vegetais, grãos integrais, nozes e produtos lácteos com baixo teor de gordura, e a restrição de sódio na presença de insuficiência cardíaca sintomática, assim como para redução da pressão arterial, tanto nos pacientes normotensos como nos hipertensos. Além disso, a redução do peso está indicada para aqueles com adiposidade corporal excessiva. Não estão explicitadas as recomendações quanto à ingestão de cada nutriente para o manejo da DCV no $\mathrm{DM}$, mas a ADA se posiciona quanto a diversas medidas dirigidas à prevenção e ao tratamento da DCV no paciente com DM (14).

O objetivo deste manuscrito foi revisar as evidências científicas nas quais se basearam as recomendações dietéticas da ADA para a prevenção e o tratamento da DCV nos pacientes com DM. Foram revisadas recomendações relacionadas apenas aos macronutrientes específicos e à perda de peso. Não foi escopo deste manuscrito a abordagem do papel dos micronutrientes da dieta no manejo da DCV nos pacientes com DM, considerando que são escassos os estudos que abordam esse tema e não haver recomendações específicas de suplementação de vitaminas e minerais para os pacientes com $\mathrm{DM}$, especialmente no que diz respeito à prevenção $\mathrm{e}$ ao tratamento da DCV.

\section{RECOMENDAÇÕES ESPECÍFICAS QUANTO AOS MACRONUTRIENTES}

\section{Gorduras}

\section{Ingestão de gorduras em geral}

A ADA recomenda ingestão de AGS de até 7\% do total de calorias, redução da ingestão de AG trans para o mínimo possível e limite de ingestão do colesterol em até $200 \mathrm{mg} /$ dia $(14,15)$. Também é recomendada a ingestão de duas ou mais porções de peixe por semana (com a exceção dos filés de peixe frito comercialmente disponíveis).

Anteriormente, a ADA recomendava que a ingestão de gordura total da dieta deveria se limitar a $30 \%$ do valor energético total (VET) (16). No entanto, vários estudos demonstraram que a quantidade total da gordura ingerida não é tão importante para a saúde cardiovascular quanto o tipo dessa gordura (17). A dieta com restrição de gordura pode ter benefício especialmente na redução do peso corporal (9). Por esse motivo, atualmente, a ADA só recomenda o limite de ingestão de até $30 \%$ de gordura total quando discute medidas dietéticas para redução do peso corporal (14).

As recomendações dietéticas atuais da ADA estão de acordo com o Third Report of the National Cholesterol Education Program (NCEP) Expert Panel on Detection, Evaluation, and Treatment of High Blood Cholesterol in Adults (Adult Treatment Panel III [ATP III]) (18). A meta principal das recomendações em relação a DCV tem sido a redução do colesterol-LDL (19). A 
partir de 2002, o DM passou a ser considerado como um equivalente de DCV estabelecida (18), sendo recomendada aos pacientes com DM uma abordagem terapêutica mais agressiva, principalmente no manejo dos lipídeos séricos, similar a dos pacientes sem DM e com doença arterial coronariana estabelecida (18). A recomendação foi baseada, fundamentalmente, em grandes ensaios clínicos aleatorizados (ECA) realizados com estatinas (18) e, nessa época, já havia evidência do benefício também nos subgrupos de pacientes com DM. Em 2004, após divulgação do Heart Protection Study (20) e do Collaborative Atorvastatin Diabetes Study (21), essas evidências foram reforçadas, principalmente para a prevenção primária de DCV nos pacientes com DM (22).

A partir de 2006, com o endosso da Associação Americana de Cardiologia (6), a ADA passou a adotar as orientações dietéticas para os pacientes com DM, quanto à ingestão de gorduras, similares às dos pacientes com DCV estabelecida (18). Também com base nessas recomendações, as metas atuais de valores de colesterol-LDL em pacientes com DM são $<100 \mathrm{mg} / \mathrm{dL}$ e, para pacientes com DM e com muito alto risco cardiovascular, $<70 \mathrm{mg} / \mathrm{dL}$ (18). Entretanto, deve ser considerado que o risco absoluto para eventos cardiovasculares não é o mesmo em todos os pacientes com $\operatorname{DM}(23,24)$ e sugere-se a utilização de escores de risco cardiovascular como, por exemplo, o UKPDS Risk Engine, validado especificamente para pacientes com DM para a prevenção e manejo da DCV (25).

É importante salientar que as intervenções dietéticas, em geral, promovem modificações no perfil lipídico plasmático de magnitude limitada. Estudos prévios em indivíduos não diabéticos já mostraram que a expectativa de redução do colesterol-LDL com as modificações na ingestão de gorduras é em torno de 15 a $25 \mathrm{mg} / \mathrm{dL}$ (26). Reduções maiores, de até $40 \%$, têm sido descritas, mas em ensaios clínicos nos quais as intervenções dietéticas foram associadas a outras abordagens sobre o estilo de vida, como redução do peso corporal e aumento da atividade física $(27,28)$. A redução dos valores de colesterol-LDL resultante de modificações dietéticas dependerá dos hábitos alimentares basais, da aderência à intervenção dietética e da resposta biológica individual.

\section{Ácidos graxos saturados e colesterol}

O AGS e o colesterol dietético aumentam o risco de DCV, em parte, por meio de efeitos nos lipídeos plasmáticos. Além disso, a gordura saturada também tem efeito pró-coagulante, reduzindo a sensibilidade à insulina (29) e está associada à disfunção endotelial (30). O colesterol proveniente da dieta aumenta o colesterol plasmático, mas seu papel regulador é menor que o dos AGS (9).

A diminuição das concentrações sanguíneas de colesterol total e LDL, obtida por meio da redução da ingestão dos AGS, com consequente redução do risco de DCV, é bem demonstrada em indivíduos não diabéticos (18). Para cada $1 \%$ de diminuição da energia proveniente dos AGS da dieta, ocorre redução do colesterol-LDL de aproximadamente $2 \%$ (31). No estudo Delta (32), a redução dos AGS de 15 para $6,1 \%$ do VET resultou em redução de colesterol-LDL em torno de $11 \%$. A magnitude da redução do colesterol-LDL promovida pela restrição no conteúdo de AGS da dieta parece ser independente do tipo de nutriente pelo qual ele é substituído, sendo os resultados semelhantes quando a substituição é por carboidratos ou por AG monoinsaturados (AGMI) (33).

Em pacientes com DM, estudos observacionais analisaram a associação entre o conteúdo de gordura da dieta e as complicações cardiovasculares. No estudo de Soinio e cols. (34), no qual foram avaliados 366 pacientes com DMT2 sem doença arterial coronariana, foi observada associação negativa entre a razão de AGPI/ AGS (razão P:S) e a taxa de morte cardiovascular entre homens, ajustada para os fatores de risco cardiovascular convencionais. A ingestão média de AGS, no início do estudo, era em torno de 19 a $20 \%$ do VET diário e sem diferença entre os pacientes que tiveram ou não mortalidade por doença arterial coronariana. Em coorte de mulheres com DM, uma dieta caracterizada por maior ingestão de colesterol $(298 \mathrm{mg} / 1.000 \mathrm{kcal}$ versus 139 $\mathrm{mg} / 1.000 \mathrm{kcal})$ e de AGS (19,1\% VET versus $10,8 \%$ VET) e baixa razão $P: S(1,0$ versus 0,66$)$ associou-se com risco aumentado de DCV (35).

A recomendação para redução da ingestão de AGS para $<7 \%$ do VET é considerada nível A de evidência pela ADA (14), tendo sido baseada em estudos que analisaram o efeito das modificações no conteúdo de AGS realizados apenas em indivíduos sem DM $(18,31,32)$ e em estudos conduzidos em pacientes com DM $(18,20,21)$, mas que avaliaram o efeito da redução do colesterol-LDL com tratamento farmacológico para fatores de risco ou desfechos cardiovasculares. Não estão disponíveis ECA que avaliem o efeito de modificações no conteúdo de gordura saturada sobre fatores de risco e/ou desfechos cardiovasculares em pacientes com DM. 
Particularidade importante é o efeito distinto dos diferentes AGS sobre os lipídeos plasmáticos (36). Por exemplo, o ácido palmítico e o mirístico da dieta aumentam o colesterol total e LDL, enquanto o ácido esteárico reduz essas lipoproteínas. Portanto, além de atentar-se à quantidade de AGS ingerido, é essencial o conhecimento sobre as fontes alimentares de cada AGou de cada um desses AGS).

\section{Ácidos graxo trans}

A ADA recomenda a redução da ingestão de AG trans para o mínimo possível $(14,15)$. Os AG trans aumentam as concentrações sanguíneas de colesterol-LDL e lipoproteína (a) e diminuem as de colesterol-HDL (37). A gordura trans também provoca aumento nos valores séricos de triglicerídeos e redução do tamanho da partícula de LDL, além de promover a inflamação, expressa pela elevação das concentrações circulantes de fator de necrose tumoral-alfa (TNF- $\alpha$ ), interleucina- 6 (L-6) e proteína C-reativa, o que mostra que o aumento desse tipo de gorduras implica disfunção endotelial e expressão de moléculas de adesão (37).

O Departamento de Agricultura Americana e a Associação Americana de Cardiologia recomendam que o consumo de gordura trans seja menor do que $1 \%$ do VET para a população geral, sem recomendações específicas para a população com DM (38). Essa orientação é baseada na evidência de que os AG trans aumentam o risco de doença arterial coronariana mais do que qualquer outro nutriente, considerando-se que esses efeitos são vistos mesmo com valores de ingestão muito baixos (1 a $3 \%$ do valor calórico total) $(8,37,38)$. Em metanálise de quatro estudos prospectivos, na qual foram avaliados em torno de 140 mil pacientes, evidenciou-se que aumento de $2 \%$ no valor energético proveniente da ingestão de gordura trans elevou em $23 \%$ a incidência de doença arterial coronariana (37).

\section{Ácidos graxo poli-insaturados}

Maior ingestão de peixe é recomendada pela $\mathrm{ADA}$ $(14,15)$, considerando ser este alimento rico em AGPI. Entre os principais AGPI provenientes da dieta estão os AGPI $\omega-6$, cujo principal representante é o ácido linoleico (18:2n6), e os AGPI $\omega$-3, dentre eles o ácido linolênico (18:3n3), os ácidos de cadeia longa eicosapentaenoico (EPA:20:5n3) e o docosa-hexaenoóico (DHA:22:6n3), além do ácido de cadeia intermediária alfa-linolênico (ALA:18:3n3) que é convertido a EPA ou DHA. O peixe tem como principais AGPI o EPA e o DHA, aos quais tem sido especialmente atribuído o efeito benéfico sobre as DCV. Entre os benefícios, destaca-se a redução na ocorrência de arritmias, nos triglicerídeos plasmáticos, nos níveis de pressão arterial, na agregação plaquetária e em marcadores inflamatórios circulantes $(34,39-41)$.

Nos indivíduos sem DM, são inúmeros os estudos mostrando o benefício da ingestão de AGPI $\omega$-3 sobre a prevenção primária e secundária de DCV. Metanálise recente de estudos observacionais e ECA analisou o efeito da maior ingestão de AGPI $\omega$-3 (suplementação na dieta ou consumo de peixe) sobre a redução de desfechos cardiovasculares em indivíduos sem DM (42). Com relação à prevenção primária de $\mathrm{DCV}$, a associação da maior ingestão de AGPI $\omega$-3, assim como do consumo de peixe, com a redução de risco de morte de origem cardíaca, morte súbita e infarto do miocárdio, foi bem evidente, mas apenas nos estudos de coorte ou caso-controle. Já para a prevenção secundária (pacientes com DCV prévia), os ECA revelaram que a ingestão dos AGPI $\omega$-3 foi associada à redução de mortalidade total, morte de origem cardíaca e infarto do miocárdio, mais bem evidenciada nos estudos cuja intervenção dietética se baseava no aumento do consumo de peixe. Em um único estudo de coorte em pacientes com doença arterial coronariana houve redução significante na mortalidade total $(\mathrm{RR}=$ 0,37 ) nos pacientes que consumiram $>57 \mathrm{~g} /$ dia de peixe. Entretanto, os estudos incluídos na referida revisão apresentam limitações: são heterogêneos em relação à estimativa da ingestão de $\omega-3$, às características basais das dietas, à presença de fatores de risco, aos tipos de peixe ingeridos (em razão da diferença no conteúdo de EPA e DHA), assim como ao método de preparo dos peixes.

Recentemente, foram publicados os resultados do estudo JELIS, ECA realizado com 18.645 pacientes hipercolesterolêmicos com e sem coronariopatia prévia (43). A intervenção consistia na suplementação de $1.800 \mathrm{mg} /$ dia de EPA com estatina, e o grupo controle recebia apenas estatina, por um período de cinco anos. Os autores observaram redução de 19\% na ocorrência de eventos coronarianos no grupo com adição de EPA. Quando os pacientes com e sem doença arterial coronariana foram analisados em separado, apenas nos primeiros houve redução de eventos coronarianos principais (19\%).

Nos pacientes com DM, os efeitos benéficos dos AGPI $\omega$-3 de cadeia longa sobre os fatores de risco cardiovascular foram bem descritos (44). A associação da ingestão de AGPI $\omega-3$ e do consumo de peixe com a 
incidência de doença arterial coronariana e mortalidade total foi avaliada em um subgrupo de enfermeiras com DMT2, participantes do Nurses' Health Study (45). Foram estudadas 5.103 pacientes sem diagnóstico inicial de doença arterial coronariana e acompanhadas por até 16 anos. Comparando as pacientes que raramente ingeriam peixe (menos de uma porção ao mês), o risco relativo (intervalo de confiança, IC, de 95\%) para o consumo de peixe uma vez por semana foi de $0,60(0,42$ a 0,85$)$; para o consumo de duas a quatro vezes por semana, de 0,64 $(0,42$ a 0,99$)$ e para o consumo de mais de cinco vezes por semana, foi de $0,36(0,20$ a 0,66$)$ ( $\mathrm{p}$ de tendência $=$ $0,002)$. O maior consumo de peixe (duas porções por semana) por um período de três anos também foi associado a menores aumentos no percentual de estenose, avaliada por meio de angiografia coronariana quantitativa, em mulheres com doença arterial coronariana e $\mathrm{DM}$, não sendo esse efeito verificado em pacientes sem DM (46).

O efeito benéfico da maior ingestão de AGPI $\omega-3$ sobre a macroangiopatia diabética foi também estudado por Mita e cols. (47), analisando o espessamento da íntima-média da carótida. Em ECA controlado, a intervenção consistiu da suplementação de $1.800 \mathrm{mg}$ de EPA a pacientes com DMT2, durante um período médio de dois anos. Os autores observaram redução significante da espessura da íntima-média da carótida, diretamente associada à suplementação do EPA.

Os AGPI em geral - e não apenas os da classe $\omega-3$ - podem ter efeitos benéficos sobre os fatores de risco cardiovasculares em pacientes com DM. Recentemente, Almeida e cols. (48) observaram associação inversa entre a ingestão de AGPI totais, especialmente os de origem vegetal, e a presença de microalbuminúria em pacientes com DMT2, condição de risco cardiovascular aumentado (49). Já a ingestão de ALA não parece ter efeitos sobre a morbimortalidade cardiovascular (42).

Para finalizar, convém ressaltar que o tipo de preparo do peixe pode afetar o conteúdo de $\omega$-3 ou ainda adicionar AGS ou AG trans. Maior ingestão de peixe não deve ocorrer à custa de filés de peixe frito comercialmente disponíveis. De fato, Mozaffarian e cols. (50) observaram tendência ao aumento do risco de eventos cardiovasculares associado à ingestão de peixe frito ou em sanduíche em indivíduos sem DCV prévia.

\section{Carboidratos}

As recomendações dietéticas da $\mathrm{ADA}$ em relação a $\mathrm{DCV}$ não incluem orientações específicas quanto ao conteúdo de carboidratos, sendo o consumo de carboidratos inserido apenas nas orientações relacionadas ao controle glicêmico e à prevenção de complicações crônicas. A ADA recomenda a ingestão de carboidratos provenientes de frutas, vegetais, grãos integrais, legumes e leite desnatado, por serem estes recomendados para uma vida saudável $(14,15)$. Os carboidratos simples, como a sacarose, devem ser substituídos por outras fontes de carboidratos, uma vez que são mais rapidamente digeridos e absorvidos do que os amidos, além de contribuírem para o excesso de energia ingerida.

Um fator a considerar em relação aos carboidratos é a resposta glicêmica à sua ingestão. Diferentes fontes de carboidrato variam quanto às suas taxas de absorção, interferindo, assim, nos seus efeitos sobre as concentrações plasmáticas de glicose e insulina. Essas variações na resposta dos carboidratos da dieta podem ser quantificadas por meio do índice glicêmico e da carga glicêmica dos alimentos. Alguns ECA têm demonstrado que dietas com baixo índice glicêmico podem reduzir a glicemia em pacientes com DM, mas outros não confirmaram esse achado (51). Com base nessas observações, a ADA recomenda levar em consideração a carga e o índice glicêmicos da dieta como um possível benefício adicional para manejo do controle glicêmico.

Considerando que a glicemia pós-prandial é um fator de risco progressivo para a DCV, tanto em indivíduos diabéticos como em não diabéticos (52), dietas com baixo índice glicêmico poderiam estar associadas ao menor risco de DCV. Metanálise recente de estudos de coorte prospectivos demonstrou benefício de dietas com baixo índice glicêmico em mulheres não diabéticas (53). $\mathrm{O}$ mesmo efeito não se confirmou nos homens não diabéticos (54). Da mesma maneira, não existe ainda evidência de que dietas com baixo índice glicêmico tragam benefício na prevenção e no tratamento das DCV em pacientes com DM.

Dietas ricas em carboidratos, predominantemente de fontes integrais, têm sido amplamente recomendadas na tentativa de se diminuir o teor de gordura saturada da dieta. Entretanto, dietas hiperglicídicas podem causar hipertrigliceridemia (induzida por carboidratos), favorecer a formação de partículas de LDL pequenas e densas, e reduzir as concentrações plasmáticas de colesterol HDL (55). Esses efeitos sobre o perfil lipídico parecem ser acentuados quando a dieta é rica em carboidratos de alto índice glicêmico, como é o caso de alguns monossacarídeos, alimentos processados e farinhas refinadas. Por outro lado, tais efeitos podem ser atenuados por dietas ricas em carboidratos com baixo índice glicêmico, tais como grãos integrais (55). Desse modo, a decisão 
de substituir gorduras por carboidratos deve levar em consideração o tipo de carboidrato escolhido.

Pequena restrição de carboidrato associada a aumento de ingestão de proteínas e AG insaturados poderia influenciar favoravelmente diversos fatores de risco cardiovascular (56). Em um ECA de 12 semanas em pacientes com DMT2, foram comparadas dietas com percentual proteico elevado ( $28 \%$ do VET) e baixo percentual de carboidratos ( $42 \%$ do VET) com dietas com alto percentual de carboidratos ( $55 \%$ do total de energia). O controle glicêmico não foi diferente nos dois tipos de dieta, mas o colesterol-LDL foi menor na dieta com elevado percentual proteico e, nas mulheres, a redução de massa gorda foi maior (57).

Finalmente, dietas pobres em carboidratos poderiam constituir uma alternativa para perda de peso. ECA recentes com esse tipo de dietas, envolvendo pacientes sem DM e com tempo de seguimento igual ou superior a 12 meses, apresentam resultados diversos. Em dois estudos ocorreu um efeito maior na perda de peso com uma dieta pobre em carboidratos, hipocalórica (58) ou não (59). Entretanto, no mais longo (24 meses) desses ECA, não houve diferença na perda de peso com a adoção de uma dieta com baixo teor de carboidratos ( $40 \%$ da energia proveniente de lipídeos, $25 \%$ de energia de proteínas e $35 \%$ de energia de carboidratos), quando comparada às dietas com maiores proporções de carboidratos (60). Já entre os pacientes com DM, também as evidências são insuficientes para fazer recomendações a favor ou contra o uso de dietas pobres em carboidratos. De acordo com a ADA, as dietas pobres em carboidratos $(<130 \mathrm{~g} /$ dia $)$ não são recomendadas no manejo do DM em razão da falta de evidência científica suficiente que suporte suas segurança e eficácia $(14,15)$.

Apesar da diversidade e da quantidade de recomendações existentes, ainda há na literatura controvérsias a respeito de qual seria a quantidade ideal de carboidrato a ser ingerida pelo paciente com DM, em especial se relacionada à prevenção da DCV. A orientação dietética quanto aos carboidratos deve ser individualizada e balanceada, baseando-se nas necessidades nutricionais recomendadas pela Dietary Reference Intakes (61). Futuros estudos sobre a composição ideal da dieta devem enfocar tanto a quantidade como a qualidade do carboidrato que deve provir da dieta.

\section{Fibras}

Com relação à ingestão de fibras, a $\mathrm{ADA}$ recomenda aumentar seu consumo, tendo como meta uma inges- tão diária de pelo menos $14 \mathrm{~g} / 1.000 \mathrm{kcal}$, similar ao recomendado para a população em geral $(14,15)$. Uma ingestão maior do que essa sugerida ainda não pode ser recomendada, por falta de evidências que comprovem benefício nos pacientes com DM.

O conteúdo de fibras da dieta tem um importante benefício sobre o controle metabólico (28). Ela atua como uma bile sequestradora de ácido, que propicia a redução do colesterol. Além do mais, a fibra parece diminuir o esvaziamento gástrico e a absorção de glicose, diminuindo, assim, o grau de resposta de insulina a uma refeição e subsequente lipogênese hepática. O conteúdo de fibra da dieta parece agir também sobre a secreção de insulina em indivíduos saudáveis, sugerindo, talvez, melhora na função das células $\beta$ em longo prazo (62). Outros possíveis benefícios também são observados sobre o controle do peso corporal e pressão arterial (63).

Em estudos observacionais, o efeito da maior ingestão de fibras sobre os fatores de risco cardiovascular são bem evidentes, tanto em pacientes sem como com DM. Utilizando a base de dados do National Health and Nutrition Examination Survey 1999-2000, King e cols. (64) observaram que os indivíduos nos terceiro e quarto quartis de consumo de fibras na dieta apresentaram um menor risco de aumento da proteína Creativa, considerado um fator de risco de $\mathrm{DCV}$ não tradicional quando comparados aos indivíduos do menor quartil.

Deve ser ressaltado que o efeito da ingestão de alimentos com elevado teor de carboidratos sobre a glicemia, assim como sobre os lipídeos séricos, depende não apenas da quantidade, mas também do tipo de fibra do alimento (65). Neste sentido, as fibras solúveis teriam uma ação benéfica mais importante sobre o perfil lipídico do que as fibras insolúveis. A ADA recomenda uma ingestão de 10 a $25 \mathrm{~g}$ de fibra solúvel por dia para prevenção de dislipidemia (15). Já o NCEP-ATP III (18) recomenda o consumo diário de 5 a $10 \mathrm{~g}$ de fibras solúveis associado a outras recomendações gerais sobre macronutrientes, com o objetivo de normalizar os elevados valores de colesterol-LDL.

Em pacientes com DMT2, Steemburgo e cols. (66) recentemente avaliaram a associação entre a ingestão de fibras e a presença de síndrome metabólica. Nesse estudo, a ingestão de fibras solúveis, particularmente provenientes de alimentos contendo grãos integrais e frutas, apresentou um papel protetor para a presença de síndrome metabólica, condição associada a um maior risco de DCV (6). 
Recentemente, foi realizada uma revisão sistemática de dez ECA e controlados, com duração de quatro a oito semanas, que analisaram a relação entre o consumo de alimentos contendo grãos integrais e o seu efeito sobre os fatores de risco cardiovascular (67). Foram estudados pacientes com pelo menos um fator de risco ou com DCV estabelecida. Foi observada redução do colesterol total e do LDL com a maior ingestão de fibras. No entanto, oito dos estudos analisaram a ingestão de aveia como fonte de fibras e o efeito sobre o perfil lipídico foi associado apenas à ingestão desses alimentos - efeito realmente esperado, já que a aveia é uma importante fonte de fibras solúveis.

O efeito da ingestão de fibras sobre os eventos coronarianos também é bem documentado. Em uma análise dos dados originais de dez estudos de coorte, observou-se uma associação inversa entre o consumo de fibras provenientes de frutas e cereais e o risco de doença arterial coronariana (68). Posteriormente, um estudo finlandês, realizado em mulheres pós-menopáusicas e com coronariopatia estabelecida, analisou a associação entre a ingestão de fibras e a progressão das alterações angiográficas coronarianas após um seguimento médio de três anos. O maior consumo de fibras provenientes de cereais e grãos integrais foi associado à menor progressão da aterosclerose coronária nessas pacientes (69).

Assim, a associação da maior ingestão de fibras na dieta, tanto com a redução dos fatores de risco cardiovasculares como com a menor progressão do processo aterosclerótico em pacientes com DM, está bem documentada em estudos observacionais e parece ser de magnitude significativa. Baseando-se nessas observações, atribui-se um nível de evidência B para recomendar um maior consumo de fibras na dieta, principalmente de fibras solúveis, nos pacientes com DM para prevenção e tratamento das DCV. ECA que analisem o efeito da ingestão de fibras sobre desfechos CV, especialmente nos pacientes com DM, poderão reforçar essa recomendação.

\section{Proteínas}

A ADA não apresenta uma recomendação específica quanto à ingestão de proteínas para o tratamento e manejo do risco para DCV. As recomendações sobre a ingestão proteica são inseridas no manejo do DM e direcionadas à nefropatia diabética. Neste sentido, considerando que os pacientes com nefropatia diabética têm elevado risco de DCV, o manejo dessa situação (prevenção ou tratamento) poderia ser inserido nas recomen- dações relativas a DCV. A manipulação do conteúdo proteico da dieta poderia ser também utilizada como estratégia para redução do risco de DCV em pacientes com sobrepeso e obesidade. Entretanto, dietas com altos valores proteicos não são indicadas para perda de peso, pois os efeitos em longo prazo desse tipo de dieta não são conhecidos. A ingestão diária de proteínas para indivíduos com DM deve ser similar à da população em geral, não excedendo $20 \%$ do VET. Recomenda-se a redução do consumo de proteínas para 0,8 a $1,0 \mathrm{~g} / \mathrm{kg}$ peso/dia para pacientes em estágios iniciais da doença renal crônica e $0,8 \mathrm{~g} / \mathrm{kg}$ peso/dia para aqueles com doença renal avançada $(14,15)$.

\section{RECOMENDAÇÃO DIETÉTICA PARA REDUÇÃO DO PESO CORPORAL}

Nos pacientes obesos com DMT2, a redução da ingestão calórica e do peso corporal propicia um aumento na sensibilidade à insulina, melhora no controle glicêmico e no perfil lipídico e redução da pressão arterial (9). Esses benefícios são alcançados já com uma redução modesta $(5 \%)$ do peso corporal (70).

Recentemente, foram publicados resultados de um ano de seguimento do estudo Look AHEAD (Action for Health in Diabetes), ensaio clínico conduzido em 16 centros americanos para determinar o efeito da redução do peso corporal em longo prazo sobre o controle glicêmico e sobre a prevenção de eventos cardiovasculares em pacientes com DMT2 (71). Os pacientes submetidos à intervenção (modificação dos hábitos alimentares e aumento da atividade física) apresentaram perda de peso média de $8,6 \%$ do peso corporal inicial. Além disso, durante o estudo, a perda de peso corporal foi associada à melhora do controle glicêmico e de fatores de risco cardiovascular: pressão arterial, hemoglobina glicosilada, triglicerídeos, colesterol-HDL e albuminúria.

$\mathrm{Na}$ estratégia da redução do peso corporal, o mais importante no que diz respeito às modificações dietéticas é a redução do valor calórico total da dieta. Deve ser ressaltado que os ECA que demonstraram $(58,59)$ ou não (60) benefícios com a perda de peso por meio de modificação de nutrientes ou do tipo de alimento não foram realizados em sua maioria em pacientes com DM.

\section{CONSIDERAÇÕES FINAIS}

Apesar de todo avanço nas propostas farmacológicas para manejo do DM e prevenção de suas complicações, 
a intervenção dietética persiste como medida primordial para prevenção primária e secundária da DCV.

As orientações dietéticas devem seguir as necessidades nutricionais determinadas pela Dietary Reference Intakes (61), assim como diretrizes desenvolvidas por sociedades científicas, cujas recomendações se baseiam em estudos disponíveis com o melhor nível de evidência possível. As diretrizes da ADA foram recentemente atualizadas quanto às recomendações dietéticas para os pacientes com DM que, na sua grande maioria, vem ao encontro das diretrizes de outras sociedades reconhecidas, como a American Heart Association, estabelecidas para indivíduos sem DM na prevenção e manejo das DCV (72).

As recomendações de aspectos da dieta da ADA para prevenção e tratamento da $\mathrm{DCV}$ no $\mathrm{DM}$, de maneira geral, são baseadas em evidências científicas de estudos com indivíduos sem DM. Na tabela 1, estão resumidas essas recomendações e os principais estudos que funda- mentam tais diretrizes. Nas situações em que não foram encontrados estudos específicos que justificassem as recomendações, foram citados consensos de entidades ou associações reconhecidas. Embora o DM seja considerado um equivalente de DCV estabelecida, essa indicação pode ser questionável conforme discutido anteriormente. Deve ser lembrado que existem aspectos peculiares da DCV nos pacientes com DM. É possível que os pacientes com DM e risco cardiovascular elevado beneficiem-se tanto ou mais do que os pacientes não diabéticos com risco cardiovascular elevado. Portanto, persiste a necessidade de confirmação de muitas dessas recomendações por meio de ECA realizados em pacientes com DM. Esses estudos permitirão que as diretrizes internacionais sejam atualizadas com vistas à prevenção e ao tratamento da DCV, especificamente no DM.

Declaração: os autores declaram não haver conflitos de interesse científico neste estudo.

Tabela 1. Evidências científicas para embasamento das recomendações dietéticas da ADA para prevenção e tratamento das doenças cardiovasculares em pacientes com diabetes melito

\begin{tabular}{|c|c|c|c|c|}
\hline & \multirow{2}{*}{ Recomendações (nível de evidência) } & & \multicolumn{2}{|c|}{ Evidências sugeridas com base em: } \\
\hline & & & Estudos em indivíduos sem DM & Estudos em pacientes com DM \\
\hline AGS & $<7 \%$ VET & C & ECA (32) & - \\
\hline Colesterol & $<200 \mathrm{mg} / \mathrm{dia}$ & B & Consenso (18) & Coorte (35) \\
\hline AG trans & Reduzir ao mínimo possível & $E$ & $\begin{array}{c}\text { Coorte (37) } \\
\text { Consenso (38) }\end{array}$ & - \\
\hline AGPI & Ingestão de 2 porções de peixe por semana & A & $\begin{array}{l}\mathrm{ECA}(42,43) \\
\text { Coorte }(42)\end{array}$ & $\begin{array}{c}\text { ECA }(47) \\
\text { Coorte }(45,46,48)\end{array}$ \\
\hline Índice glicêmico & Preferência por carboidratos com baixo índice glicêmico & C & $\begin{array}{c}\text { Coorte (53) } \\
\text { Consenso (55) }\end{array}$ & Consenso (55) \\
\hline Fibras & $\begin{array}{l}14 \mathrm{~g} / 1.000 \text { calorias } \\
\text { (fibra solúvel = } 5 \mathrm{a} 10 \mathrm{~g} / \mathrm{dia} \text { ) }\end{array}$ & B & $\begin{array}{c}\operatorname{ECA}(67) \\
\text { Coorte }(18,64,67,69)\end{array}$ & Coorte (66) \\
\hline Peso corporal & Se sobrepeso ou obesidade, redução moderada ( $5 \%$ a $7 \%$ ) & A & $\operatorname{ECA~}(58,59,60)$ & ECA (71) \\
\hline
\end{tabular}

DM: diabetes melito; AGS: ácidos graxos saturados; VET: valor energético total; ECA: ensaio clínico aleatorizado; AGPI: ácidos graxos poli-insaturados.

\section{REFERÊNCIAS}

1. Thom T, Haase N, Rosamond W, Howard VJ, Rumsfeld J, Manolio T, Zheng ZJ, Flegal K, O'Donnell C, Kittner S, Lloyd-Jones D, Goff DC Jr, HongY, Adams R, Friday G, Furie K, Gorelick P, Kissela B, Marler J, Meigs J, Roger V, Sidney S, Sorlie P, Steinberger J, Wasserthiel-Smoller S, Wilson M, Wolf P; American Heart Association Statistics Committee and Stroke Statistics Subcommittee. Heart disease and stroke statistics-2006 update: a report from the American Heart Association Statistics Committee and Stroke Statistics Subcommittee. Circulation. 2006;113(6):e85-151.

2. United Kingdom Prospective Diabetes Study Group. UKPDS 17. A nineyear update of a randomized, controlled trial on the effect of improved metabolic control on complications in noninsulindependent diabetes mellitus. Ann Intern Med. 1996;124(1SII):13645.
3. Scheffel RS, Bortolanza D, Weber CS, Costa LA, Canani LH, Santos $\mathrm{KG}$, et al. Prevalence of micro and macroangiopatic chronic complications and their risk factors in the care of out patients with type 2 diabetes mellitus. Rev Assoc Med Bras. 2004;50(3):263-7.

4. Bloomgarden ZT. Cardiovascular disease and diabetes. Diabetes Care. 2003; 26(1):230-7.

5. Turner RC, Millns H, Neil HA, Stratton IM, Manley SE, Matthews $\mathrm{DR}$, et al. Risk factors for coronary artery disease in non-insulin dependent diabetes mellitus: United Kingdom Prospective Diabetes Study (UKPDS 23). BMJ. 1998;316(7134):823-8.

6. Wilson PW, D'Agostino RB, Parise H, Sullivan L, Meigs JB. Metabolic syndrome as a precursor of cardiovascular disease and type 2 diabetes mellitus. Circulation. 2005;112(20):3066-72.

7. Picon PX, Zanatta CM, Gerchman F, Zelmanovitz T, Gross JL, Canani LH. Análise dos critérios de definição da síndrome metabóli- 
ca em pacientes com diabetes melito tipo 2. Arq Bras Endocr Metab. 2006;50(2):264-70.

8. Buse JB, Ginsberg HN, Bakris GL, Clark NG, Costa F, Eckel R, Fonseca V, Gerstein HC, Grundy S, Nesto RW, Pignone MP, Plutzky J, Porte D, Redberg R, Stitzel KF, Stone NJ; American Heart Association; American Diabetes Association. Primary prevention of cardiovascular diseases in people with diabetes mellitus: a scientific statement from the American Heart Association and the American Diabetes Association. Circulation. 2007;115(1):114-26.

9. Cernea S, Hâncu N, Raz I. Diet and coronary heart disease in diabetes. Acta Diabetol. 2003;40 Suppl 2:S389-400.

10. Hu FB, Willett WC. Optimal diets for prevention of coronary heart disease. JAMA. 2002;288(20):2569-78.

11. Mann JL. Diet and risk of coronary heart disease and type 2 diabetes. Lancet. 2002;360(9335):783-9.

12. Sacks FM, Katan M. Randomized clinical trials on the effects of dietary fat and carbohydrate on plasma lipoproteins and cardiovascular disease. Am J Med. 2002;113 Suppl 9B:13S-24S.

13. Ascherio $A$. Epidemiologic studies on dietary fats and coronary heart disease. Am J Med. 2002:113 Suppl 9B:9S-12S.

14. Bantle JP, Wylie-Rosett J, Albright AL, Apovian CM, Clark NG, Franz MJ, et al. Nutrition recommendations and interventions for diabetes - 2006: a position statement of the American Diabetes Association. Diabetes Care. 2006;29(9):2140-57.

15. American Diabetes Association. Standards of medical care in diabetes - 2009. Diabetes Care. 200932(Suppl 1):S13-S61.

16. American Diabetes Association. Nutrition principles and recommendations in Diabetes - 2004. Diabetes Care. 2004;27(Supl 1):S36-S46.

17. Howard BV, Van Horn L, Hsia J, Manson JE, Stefanick ML, Wassertheil-Smoller $\mathrm{S}$, et al. Low-fat dietary pattern and risk of cardiovascular disease: the Women's Health Initiative Randomized Controlled Dietary Modification Trial. JAMA. 2006;295(6):655-66.

18. Third Report of the National Cholesterol Education Program (NCEP) Expert Panel on Detection, Evaluation, and Treatment of High Blood Cholesterol in Adults (Adult Treatment Panel III) final report Third Report of the National Cholesterol Education Program (NCEP) Expert Panel on Detection, Evaluation, and Treatment of High Blood Cholesterol in Adults (Adult Treatment Panel III) final report. Circulation. 2002;106:3143-421.

19. Carleton RA, Dwyer J, Finberg L, Flora J, Goodman DS, Grundy SM, et al. Report of the Expert Panel on Population Strategies for Blood Cholesterol Reduction. A statement from the National Cholesterol Education Program, National Heart, Lung, and Blood Institute, National Institutes of Health. Circulation. 1991;83(6):2154-232.

20. Heart Protection Study Collaborative Group. MRC/BHF Heart Protection Study of cholesterol lowering with simvastatin in 20,536 high-risk individuals: a randomised placebo-controlled trial. Lancet. 2002;360(9326):7-22.

21. Colhoun HM, Betteridge DJ, Durrington PN, Hitman GA, Neil HA, Livingstone SJ, et al. Primary prevention of cardiovascular disease with atorvastatin in type 2 diabetes in the Collaborative Atorvastatin Diabetes Study (CARDS): multicentre randomised placebo-controlled trial. Lancet. 2004;364(9435):685-96.

22. Grundy SM, Cleeman JI, Merz CN, Brewer HB Jr, Clark LT, Hunninghake DB, et al. Implications of recent clinical trials for the National Cholesterol Education Program Adult Treatment Panel III guidelines. Circulation. 2004;110(2):227-39.

23. Evans JM, Wang J, Morris AD. Comparison of cardiovascular risk between patients with type 2 diabetes and those who had had a myocardial infarction: cross sectional and cohort studies. BMJ. 2002;324(7343):939-42.

24. Dora JM, Kramer CK, Canani LH. Standards of Medical Care in Diabetes--2008: response to Hirsch, Inzucchi, and Kirkman. Diabetes Care. 2008;31(5):e44; author reply e45.
25. Stevens RJ, Kothari V, Adler Al, Stratton IM; United Kingdom Prospective Diabetes Study (UKPDS) Group. The UKPDS risk engine: a model for the risk of coronary heart disease in Type II diabetes (UKPDS 56). Clin Sci (Lond). 2001;101(6):671-9.

26. Yu-Poth S, Zhao G, Etherton T, Naglak M, Jonnalagadda S, KrisEtherton PM. Effects of the National Cholesterol Education Program's Step I and Step II dietary intervention programs on cardiovascular disease risk factors: a metaanalysis. Am J Clin Nutr. 1999;69(4):632-46.

27. Ornish D, Scherwitz LW, Billings JH, Brown SE, Gould KL, Merritt $A$, et al. Intensive lifestyle changes for reversal of coronary heart disease. JAMA. 1998;280(23):2001-7.

28. Zarraga IGE, Schwarz ER. Impact of dietary patterns and interventions on cardiovascular health. Circulation. 2006;114(9):961-73.

29. Summers LKM, Fielding BA, Bradshaw HA, Ilic V, Beysen C, Clark $M L$, et al. Substituting dietary saturated fat with polyunsaturated fat changes abdominal fat distribution and improves insulin sensitivity. Diabetologia. 2002;45(3):369-77.

30. Perassolo MS, Almeida JC, Steemburgo T, Dall'Alba V, de Mello VD, Zelmanovitz T, et al. Endothelial dysfunction and serum fatty acid composition in patients with type 2 diabetes mellitus. Metabolism. 2008;57(9):1167-72.

31. Mensink RP, Katan MB. Effects of dietary fatty acids on serum lipids and lipoproteins: a meta-analysis of 27 trials. Arterioscler Thromb. 1992;12(8):911-9.

32. Ginsberg HN, Kris-Etherton P, Dennis B, Elmer PJ, Ershow A, Lefevre $M$, et al. Effects of reducing dietary saturated fatty acids on plasma lipids and lipoproteins in healthy subjects: the Delta Study, Protocol 1. ArteriosclerThromb Vasc Biol. 1998;18(3):441-9.

33. Garg A, Bantle JP, Henry RR, Coulston AM, Griver KA, Raatz SK, et al. Effects of varying carbohydrate content of diet in patients with non-insulin-dependent diabetes mellitus. JAMA. 1994; 271(18):1421-8.

34. Soinio M, Laakso M, Lehto S, Hakala P, Rönnemaa T. Dietary fat predicts coronary heart disease events in subjects with type 2 diabetes. Diabetes Care. 2003,26(3):619-24.

35. Tanasescu M, Cho E, Manson JE, Hu FB. Dietary fat and cholesterol and the risk of cardiovascular disease among women with type 2 diabetes. Am J Clin Nutr. 2004;79(6):999-1005.

36. Hu FB, Manson JE, Willett WC. Types of dietary fat and risk of coronary heart disease: a critical review. J Am Coll Nutr. 2001;20(1):5-19.

37. Mozaffarian D, Katan MB, Ascherio A, Stampfer MJ, Willet WC. Trans fatty acids and cardiovascular disease. N Engl J Med. 2006; 354:1601-13.

38. Department of Health and Human Services, Department of Agriculture [Internet]. Dietary guidelines for Americans 2005 (Cited 2009 Jan). Available from: http://www.health.gov/DietaryGuidelines.

39. Nair SS, Leitch JW, Falconer J, Garg ML. Prevention of cardiac arrhythmia by dietary (n_3) polyunsaturated fatty acids and their mechanism of action. J Nutr. 1997;127(3):383-93.

40. Appel LJ, Miller ER 3rd, Seidler AJ, Whelton PK. Does supplementation of diet with 'fish oil' reduce blood pressure? A meta-analysis of controlled clinical trials. Arch Intern Med. 1993;153(12):1429-38.

41. Kristensen SD, Iversen AM, Schmidt EB. $n-3$ polyunsaturated fatty acids and coronary thrombosis. Lipids. 2001;36Suppl:S79-82.

42. Wang $C$, Harris WS, Chung M, Lichtenstein AH, Balk EM, Kupelnick B, et al. n-3 Fatty acids from fish or fish-oil supplements, but not alpha-linolenic acid, benefit cardiovascular disease outcomes in primary- and secondary-prevention studies: a systematic review. Am J Clin Nutr. 2006;84(1):5-17.

43. Yokoyama $M$, Origasa $H$, Matsuzaki $M$, Matsuzawa $Y$, Saito $Y$, Ishikawa Y, Oikawa S, Sasaki J, Hishida H, Itakura H, Kita T, Kitabatake A, Nakaya N, Sakata T, Shimada K, Shirato K; Japan EPA lipid intervention study (JELIS) Investigators. Effects of eicosap- 
entaenoic acid on major coronary events in hypercholesterolaemic patients (JELIS): a randomised open-label, blinded endpoint analysis. Lancet. 2007;369(9567):1090-8.

44. Connor WE. Diabetes, fish oil, and vascular disease. Ann Intern Med. 1995;123(12):950-62.

45. Hu FB, Cho E, Rexrode KM, Albert CM, Manson JE. Fish and long-chain omega-3 fatty acid intake and risk of coronary heart disease and total mortality in diabetic women. Circulation. 2003;107(14):1852-7.

46. Erkkilä AT, Lichtenstein AH, Mozaffarian D, Herrington DM. Fish intake is associated with a reduced progression of coronary artery atherosclerosis in postmenopausal women with coronary artery disease. Am J Clin Nutr. 2004;80(3):626-32.

47. Mita T, Watada H, Ogihara T, Nomiyama T, Ogawa O, Kinoshita J, et al. Eicosapentaenoic acid reduces the progression of carotid intima-media thickness in patients with type 2 diabetes. Atherosclerosis. 2007;191(1):162-7.

48. Almeida JC, Zelmanovitz T, Vaz JS, Steemburgo T, Perassolo MS, Gross JL, et al. Sources of protein and polyunsaturated fatty acids of the diet and microalbuminuria in type 2 diabetes mellitus. J Am Coll Nutr. 2008;27(5):528-37.

49. Gross JL, Azevedo MJ, Silveiro SP, Canani LH, Caramori ML, Zelmanovitz T. Diabetic nephropathy: diagnosis, prevention, and treatment. Diabetes Care. 2005;28(1):164-76.

50. Mozaffarian D, Longstreth WT Jr, Lemaitre RN, ManolioTA, Kuller $\mathrm{LH}$, Burke GL, et al. Fish consumption and stroke risk in elderly individuals: the cardiovascular health study. Arch Intern Med. 2005;165(2):200-6.

51. Sheard NF, Clark NG, Brand-Miller JC, Franz MJ, Pi-Sunyer FX, Mayer-Davis E, et al. Dietary carbohydrate (amount and type) in the prevention and management of diabetes: a statement of the American Diabetes Association. Diabetes Care. 2004;27(9):2266-71.

52. Giugliano D, Ceriello A, Esposito K. Glucose metabolism and hyperglycemia. Am J Clin Nutr. 2008;87(1):217S-22S.

53. Barclay AW, Petocz P, McMillan-Price J, Flood VM, Prvan T, Mitchell $P$, et al. Glycemic index, glycemic load, and chronic disease risk - a meta-analysis of observational studies. Am J Clin Nutr. 2008;87(3):627-37.

54. Hare-Bruun H, Nielsen BM, Grau K, Oxlund AL, Heitmann BL. Should glycemic index and glycemic load be considered in dietary recommendations? Nutr Rev. 2008;66(10):569-90.

55. Krauss RM, Eckel RH, Howard BV, Appel LJ, Daniels SR, Deckelbaum RJ, et al. AHA Dietary Guidelines. Revision 2000: a statement for healthcare professionals from the Nutrition Committee of the American Heart Association. Circulation. 2000;102(18):2284-99.

56. Mann J, McAuley K. Carbohydrates: is the advice to eat less justified for diabetes and cardiovascular health? Curr Opin Lipidol. 2007;18(1):9-12.

57. Parker B, Noakes M, Luscombe N, Clifton P. Effect of a highprotein, high-monounsaturated fat weight loss diet on glycemic control and lipid levels in type 2 diabetes. Diabetes Care. 2002;25(3):425-30

58. Shai I, Schwarzfuchs D, Henkin Y, Shahar DR, Witkow S, Greenberg I, et al. Weight loss with a low-carbohydrate, Mediterranean, or low-fat diet. N Engl J Med. 2008;359(3):229-41.
59. Gardner CD, Kiazand A, Alhassan S, Kim S, Stafford RS, Balise RR, et al. Comparison of the Atkins, Zone, Ornish, and LEARN diets for change in weight and related risk factors among overweight premenopausal women: the ATO Z Weight Loss Study: a randomized trial. JAMA. 2007;297(9):969-77.

60. Sacks FM, Bray GA, Carey VJ, Smith SR, Ryan DH, Anton SD, et al. Comparison of weight-loss diets with different compositions of fat, protein, and carbohydrates. N Engl J Med. 2009;360(9):859-73.

61. Institute of Medicine: Dietary reference intakes: energy, carbohydrate, fiber, fat, fatty acids, cholesterol, protein, and amino acids. Washington, DC: National Academies Press; 2002.

62. Juntunen KS, Laaksonen DE, Poutanen KS, Niskanen LK, Mykkänen HM. High-fiber rye bread and insulin secretion and sensitivity in healthy postmenopausal women. Am J Clin Nutr. 2003;77(2):385-91.

63. Pereira MA, Pins JJ. Dietary fiber and cardiovascular disease: experimental and epidemiologic advances. Curr Atheroscler Rep. 2000;2(6):494-502.

64. King DE, Egan BM, Geesey ME. Relation of dietary fat and fiber to elevation of C-reactive protein. Am J Cardiol. 2003;92(11):1335-9.

65. Schulze MB, Hu FB. Dietary approaches to prevent the metabolic syndrome. Quality versus quantity of carbohydrates. Diabetes Care. 2004;27(2):613-4.

66. Steemburgo T, Dall'Alba V, Almeida JC, Zelmanovitz T, Gross JL, de Azevedo MJ. Intake of soluble fibers has a protective role for the presence of metabolic syndrome in patients with type 2 diabetes. Eur J Clin Nutr. 2009;63(1):127-33.

67. Kelly SA, Summerbell CD, Brynes A, Whittaker V, Frost G. Wholegrain cereals for coronary heart disease. Cochrane Database Syst Rev. 2007;2:CD005051.

68. Pereira MA, O'Reilly E, Augustsson K, et al. Dietary fiber and risk of coronary heart disease: a pooled analysis of cohort studies. Arch Intern Med. 2004;164(4):370-6.

69. Erkkilä AT, Herrington DM, Mozaffarian D, Lichtenstein AH. Cereal fiber and whole-grain intake are associated with reduced progression of coronary-artery atherosclerosis in postmenopausal women with coronary artery disease. Am Heart J. 2005;150(1):94-101.

70. Klein S, Sheard NF, Pi-Sunyer X, Daly A, Wylie-Rosett J, Kulkarni K, Clark NG; American Diabetes Association; North American Association for the Study of Obesity; American Society for Clinical Nutrition. Weight management through lifestyle modification for the prevention and management of type 2 diabetes: rationale and strategies: a statement of the American Diabetes Association, the North American Association for the Study of Obesity, and the American Society for Clinical Nutrition. Diabetes Care. 2004;27(8):2067-73.

71. Pi-Sunyer $X$, Blackburn G, Brancati FL, Bray GA, Bright R, Clark JM. The Look AHEAD Research Group. Reduction in weight and cardiovascular disease risk factors in individuals with type 2 diabetes. One-year results of the Look AHEAD trial. Diabetes Care. 2007;30(6):1374-83.

72. American Heart Association Nutrition Committee, Lichtenstein AH, Appel LJ, Brands M, Carnethon M, Daniels S, et al. Diet and lifestyle recommendations revision 2006: a scientific statement from the American Heart Association Nutrition Committee. Circulation. 2006;114(1):82-96. 